\title{
A Nonlinear Robust Controller Design of Lower-Triangular Systems Based on Dissipation Theory
}

\author{
Bing Wang and Yue Yuan \\ College of Energy and Electrical Engineering, Hohai University, Nanjing 211100, China \\ Correspondence should be addressed to Bing Wang; icekingking@ustc.edu
}

Received 16 May 2013; Revised 11 July 2013; Accepted 21 July 2013

Academic Editor: Baoyong Zhang

Copyright (C) 2013 B. Wang and Y. Yuan. This is an open access article distributed under the Creative Commons Attribution License, which permits unrestricted use, distribution, and reproduction in any medium, provided the original work is properly cited.

\begin{abstract}
Based on dissipation theory, a novel robust control is proposed for the lower-triangular nonlinear systems, which include strictfeedback systems and high-order lower-triangular systems. Some important concepts in dissipation theory are integrated into the recursive design, which are used to dominate the uncertain disturbance and construct the robust controller. The gotten controller renders the closed-loop system finite-gain $L_{2}$ stable in the presence of disturbance and asymptotically stable in the absence of disturbance. Especially, the controller has its advantage in regulating large disturbance. Finally, one example and one application are given to show the effectiveness of the design method. Moreover, by comparing with another robust controller, the characteristic of the proposed controller is illustrated in the simulations.
\end{abstract}

\section{Introduction}

Over the last decade, the lower-triangular systems are researched widely in the field of nonlinear systems [1-4]. This class of systems is not only important theoretically, but a lot of practical systems can conform to or be transformed into its form, such as the power generators [5], aircraft control system [6], and mobile robots [7]. It is well known that backstepping design $[8,9]$ is proposed to design the strict-feedback systems, which hold the simplest triangular structure. For different types of triangular systems, the condition for existence of control laws is investigated in [10, 11], and the explicit constructions of controllers are provided in $[12,13]$. Then, a power integrator technique is given to design the high-order lower-triangular systems, which are neither feedback linearizable nor affine in the control input $[4,14]$. Moreover, the robust control problem of lowertriangular systems has attracted a lot of attention [15-18] for overcoming the hurdle of disturbance, which possibly comes from model simplification, external disturbance, or other unknown factors.

In this paper, based on dissipation theory, a novel design method is proposed to solve the robust control problem of lower-triangular nonlinear systems in the presence of uncertain disturbance. This approach constructs the storage function and designs the controller recursively, which ensures that the closed-loop system satisfies the dissipation inequality having $L_{2}$-gain performance. The technique is firstly used in the strict-feedback system. Then, it is extended to the high-order lower-triangular system. Finally, one example of lower-triangular system and one application of synchronous generator system are given to illustrate the effectiveness of the robust control.

For the lower-triangular system with uncertain disturbance, this work uses the frame of dissipation property theory to solve the problem of disturbance rejection. First of all, the appropriate function of supply rate is chosen, which is used to gather the disturbance inputs in the recursive design. Next, the storage function and robust controller are constructed recursively to guarantee that the result at each step satisfies the dissipation property. During the design, by using feedback domination technique $[4,14]$, the disturbed parts are dominated by the functions of system states and disturbances. Then, the function of disturbances is regarded as the input of supply rate, which satisfies the dissipation inequality ensuring finite-gain $L_{2}$ stability. The effect of this method can be summarized as two aspects. Firstly, the restriction of uncertain disturbance is relaxed, and the only assumption of uncertain disturbance is bounded. 
So, no information about disturbance is required in the gotten control law, which means that certain controller can regulate uncertain disturbance. Secondly, compared with some previous robust controllers only applicable for small disturbances, the proposed robust controller is effective for all cases of bounded disturbance, especially for large disturbance.

The structure of this paper is as follows: Section 2 describes the problem and offers key lemma; Section 3 gives the robust controller design for strict-feedback systems; Section 4 extends the design method to high-order lowertriangular systems; Section 5 provides an example and application to illustrate the effectiveness of design approach. The conclusion is contained in Section 6.

\section{Problem Formulation and Key Lemma}

In this paper, we focus on the problem of constructing robust controller for the lower-triangular system, which is described by the following differential equations:

$$
\begin{aligned}
\dot{x}_{1} & =f_{1}\left(x_{1}\right)+x_{2}^{p_{1}}+\omega_{1}(t) \\
\dot{x}_{2} & =f_{2}\left(X_{2}\right)+x_{3}^{p_{2}}+\omega_{2}(t) \\
& \vdots \\
\dot{x}_{n} & =f_{n}\left(X_{n}\right)+u^{p_{n}}+\omega_{n}(t) \\
y & =x_{1},
\end{aligned}
$$

where for $i=1,2, \ldots, n$, the state $X_{i}=\left(x_{1}, \ldots, x_{i}\right)^{T} \in \mathbb{R}^{i}$, the input $u \in \mathbb{R}$, the output $y \in \mathbb{R}$, the uncertain disturbance vector $\omega=\left[\omega_{1}, \omega_{2}, \ldots, \omega_{n}\right]^{T}, \omega_{i}(t)$ is unknown nonlinear function, which denotes external disturbance with unknown bound, $f_{i}: \mathbb{R}^{i} \mapsto \mathbb{R}$ is a smooth function, and $f_{i}(0)=0$. About the system (1), one hypothesis is given as follows.

Assumption 1. $p_{i} \geq 1(i=1,2, \ldots, n)$ is odd integer, and $p_{1}$ is maximum of them.

Compared with the corresponding assumption in $[4,14]$, Assumption 1 relaxes the condition of $p_{i}$. Next, the main definitions of dissipation theory are given as follows.

Definition 2 (see [1]). The general systems are described in the following form:

$$
\begin{aligned}
\dot{x}(t) & =f\left(x, u_{0}\right) \\
y_{0}(t) & =h\left(x, u_{0}\right),
\end{aligned}
$$

where the state $x \in \mathbb{R}^{n}$, the input $u_{0} \in \mathbb{R}^{p}$, and the output $y_{0} \in \mathbb{R}^{q}$. Let the function $s\left(u_{0}, y_{0}\right): \mathbb{R}^{p} \times \mathbb{R}^{q} \mapsto \mathbb{R}$ if there exists $V(x) \geq 0, V: \mathbb{R}^{n} \mapsto \mathbb{R}^{+}$, such that

$$
V(x(\tau)) \leq V(x(0))+\int_{0}^{\tau} s\left(u_{0}, y_{0}\right) d t
$$

for any $x(0)$ and $\tau$, the system (3) is dissipative about $s\left(u_{0}, y_{0}\right)$. And $s\left(u_{0}, y_{0}\right)$ is the supply rate, and $V(x)$ is the storage function.
Remark 3. The supply rate $s\left(u_{0}, y_{0}\right)$ in Definition 2 is selectable. And there are some optional functions. The most commonly used are two options: one is $s\left(u_{0}, y_{0}\right)=u_{0}^{T} y_{0}$, and the other is $s\left(u_{0}, y_{0}\right)=\Upsilon^{2}\left\|u_{0}\right\|^{2}-\left\|y_{0}\right\|^{2}, \Upsilon>0$. In this paper, when the uncertain disturbance $\omega=\left[\omega_{1}, \omega_{2}, \ldots, \omega_{n}\right]^{T}$ is regarded as the input of the system (3), the latter is chosen as the supply rate. And if the closed-loop system is dissipative, we have

$$
V(x(\tau))-V(x(0)) \leq \int_{0}^{\tau}\left(\Upsilon^{2}\|\omega\|^{2}-\left\|y_{0}\right\|^{2}\right) d t .
$$

That is, the gain of the system is not more than $\Upsilon$ for the uncertain disturbance.

Definition 4 (see [1]). A mapping $H: L_{e}^{m} \rightarrow L_{e}^{q}$ is finite-gain $L$ stable if there exist nonnegative constants $\Upsilon_{L}$ and $\beta_{L}$, such that

$$
\left\|(H v)_{\tau}\right\|_{L} \leq \Upsilon_{L}\left\|v_{\tau}\right\|_{L}+\beta_{L}
$$

for $v \in L_{e}^{m}$ and $\tau \in[0, \infty)$.

Young's inequality is an important tool used in the recursive design, and Lemma 6 is one direct consequence of Young's inequality.

Lemma 5 (Young's inequality). For any two vectors $\bar{x}$ and $\bar{y}$, the following holds

$$
\bar{x}^{T} \bar{y} \leq \frac{\varepsilon^{p}}{p}\|\bar{x}\|^{p}+\frac{1}{q \varepsilon^{q}}\|\bar{y}\|^{q},
$$

where $\varepsilon>0$ and the constants $p>1$ and $q>1$ satisfy $(p-$ $1)(q-1)=1$.

Lemma 6 (see [19]). For real numbers $a \geq 0, b \geq 0$, and $m \geq$ 1 , the following inequality holds:

$$
a \leq b+\left(\frac{a}{m}\right)^{m}\left(\frac{m-1}{b}\right)^{m-1} .
$$

\section{Robust Controller Design of Strict-Feedback Systems}

The strict-feedback system is described by the following equations

$$
\begin{aligned}
\dot{x}_{1} & =f_{1}\left(x_{1}\right)+x_{2}+\omega_{1}(t) \\
\dot{x}_{2} & =f_{2}\left(X_{2}\right)+x_{3}+\omega_{2}(t) \\
& \vdots \\
\dot{x}_{n} & =f_{n}\left(X_{n}\right)+u+\omega_{n}(t) \\
y & =x_{1} .
\end{aligned}
$$

Based on the dissipation theory, the novel robust controller design is proposed step by step. For $i=1,2, \ldots, n$, firstly set the design parameter $C_{i}>0$. 
Step 1. Let $z_{1}=x_{1}$. The storage function is constructed as $V_{1}=z_{1}^{2} / 2$, whose derivative is

$$
\dot{V}_{1}=z_{1} \dot{z}_{1}=z_{1}\left(f_{1}\left(x_{1}\right)+x_{2}\right)+z_{1} \omega_{1}
$$

For the above equation, according to the technique of adding one power integrator $[4,14]$, by using Young's inequality (6) with $p=q=2$ and $\varepsilon=\sqrt{n} / \gamma$, the term $z_{1} \omega_{1}$ is dominated by $\left(n / 2 \gamma^{2}\right) z_{1}^{2}+\left(\gamma^{2} / 2 n\right) \omega_{1}^{2}$, where the disturbance attenuation coefficient $\gamma>0$. Taking it into formula (9) yields

$$
\dot{V}_{1} \leq z_{1}\left[x_{2}+f_{1}\left(x_{1}\right)+\left(\frac{1}{2}+\frac{n}{2 \gamma^{2}}\right) z_{1}\right]+\frac{\gamma^{2}}{2 n} \omega_{1}^{2}-\frac{z_{1}^{2}}{2} .
$$

Design the smooth virtual control as

$$
\alpha_{2}=-C_{1} z_{1}-f_{1}\left(x_{1}\right)-\frac{1}{2}\left(1+\frac{n}{\gamma^{2}}\right) z_{1}
$$

Taking controller (11) into formula (10) results in

$$
\dot{V}_{1} \leq-C_{1} z_{1}^{2}+z_{1}\left(x_{2}-\alpha_{2}\right)+\frac{\gamma^{2}}{2 n} \omega_{1}^{2}-\frac{1}{2} z_{1}^{2}
$$

Step $k$. Through $k-1$ steps, a group of virtual controllers are $z_{1}=x_{1}, z_{2}=x_{2}-\alpha_{2}, \ldots, z_{k-1}=x_{k-1}-\alpha_{k-1}$, and the storage function is $V_{k-1}=\sum_{i=1}^{k-1}\left(z_{i}^{2} / 2\right)$, whose derivative is

$$
\dot{V}_{k-1} \leq-\sum_{i=1}^{k-1} C_{i} z_{i}^{2}+z_{k-1}\left(x_{k}-\alpha_{k}\right)+\frac{\gamma^{2}}{2} \sum_{j=1}^{k-1} \frac{k-j}{n+1-j} \omega_{j}^{2}-\frac{z_{1}^{2}}{2}
$$

Let $z_{k}=x_{k}-\alpha_{k}$, and the storage function is changed into $V_{k}=V_{k-1}+z_{k}^{2} / 2$; its derivative is obtained as

$$
\begin{aligned}
\dot{V}_{k}= & \dot{V}_{k-1}+z_{k} \dot{z}_{k} \\
\leq- & -\sum_{i=1}^{k-1} C_{i} z_{i}^{2}+\frac{\gamma^{2}}{2} \sum_{j=1}^{k-1} \frac{k-j}{n+1-j} \omega_{j}^{2}-\frac{z_{1}^{2}}{2}+z_{k} \omega_{k} \\
& -z_{k} \sum_{m=1}^{k-1} \frac{\partial \alpha_{k}}{\partial x_{m}} \omega_{m}+z_{k}\left[f_{k}\left(X_{k}\right)+x_{k+1}+z_{k-1}\right. \\
& \left.-\sum_{m=1}^{k-1} \frac{\partial \alpha_{k}}{\partial x_{m}}\left(f_{m}\left(X_{m}\right)+x_{m+1}\right)\right] .
\end{aligned}
$$

By using Young's inequality, we have

$$
\begin{aligned}
\dot{V}_{k} \leq & -\sum_{i=1}^{k-1} C_{i} z_{i}^{2}+\frac{\gamma^{2}}{2} \sum_{j=1}^{k-1} \frac{k-j}{n+1-j} \omega_{j}^{2}-\frac{z_{1}^{2}}{2} \\
& +\frac{\gamma^{2}}{2(n+1-k)} \omega_{k}^{2}+\frac{\gamma^{2}}{2} \sum_{m=1}^{k-1} \frac{\omega_{m}^{2}}{n+1-m}
\end{aligned}
$$

$$
\begin{gathered}
+z_{k}\left[f_{k}\left(X_{k}\right)+x_{k+1}+z_{k-1}+\frac{n+1-k}{2 \gamma^{2}} z_{k}\right. \\
+\frac{z_{k}}{2 \gamma^{2}} \sum_{m=1}^{k-1}(n+1-m)\left(\frac{\partial \alpha_{k}}{\partial x_{m}}\right)^{2} \\
\left.-\sum_{m=1}^{k-1} \frac{\partial \alpha_{k}}{\partial x_{m}}\left(f_{m}\left(X_{m}\right)+x_{m+1}\right)\right] .
\end{gathered}
$$
follows:

In this step, the smooth virtual controller is taken as

$$
\begin{aligned}
\alpha_{k+1}= & -C_{k} z_{k}-f_{k}\left(X_{k}\right)-z_{k-1}-\frac{n+1-k}{2 \gamma^{2}} z_{k} \\
& -\frac{z_{k}}{2 \gamma^{2}} \sum_{m=1}^{k-1}(n+1-m)\left(\frac{\partial \alpha_{k}}{\partial x_{m}}\right)^{2} \\
& +\sum_{m=1}^{k-1} \frac{\partial \alpha_{k}}{\partial x_{m}}\left(f_{m}\left(X_{m}\right)+x_{m+1}\right) .
\end{aligned}
$$

Under the action of the above controller, formula (15) is turned into

$$
\dot{V}_{k} \leq-\sum_{i=1}^{k} C_{i} z_{i}^{2}+z_{k}\left(x_{k+1}-\alpha_{k+1}\right)+\frac{\gamma^{2}}{2} \sum_{j=1}^{k} \frac{k+1-j}{n+1-j} \omega_{j}^{2}-\frac{z_{1}^{2}}{2} .
$$

Step $n$. Let $z_{n}=x_{n}-\alpha_{n}$, and the storage function is $V=V_{n}=$ $V_{n-1}+z_{n}^{2} / 2=(1 / 2) \sum_{i=1}^{n} z_{i}^{2}$. The nonlinear robust controller is designed as

$$
\begin{aligned}
u= & -C_{n} z_{n}-f_{n}\left(X_{n}\right)-z_{n-1}-\frac{z_{n}}{2 \gamma^{2}} \\
& -\frac{z_{n}}{2 \gamma^{2}} \sum_{m=1}^{n-1}(n+1-m)\left(\frac{\partial \alpha_{n}}{\partial x_{m}}\right)^{2} \\
& +\sum_{m=1}^{n-1} \frac{\partial \alpha_{n}}{\partial x_{m}}\left(f_{m}\left(X_{m}\right)+x_{m+1}\right) .
\end{aligned}
$$

Now, the derivative of the storage function is

$$
\dot{V}_{n} \leq-\sum_{i=1}^{n} C_{i} z_{i}^{2}+\frac{\gamma^{2}}{2} \sum_{j=1}^{n} \omega_{j}^{2}-\frac{z_{1}^{2}}{2} .
$$

From system (8), it is known that the uncertain disturbance vector $\omega=\left[\omega_{1}, \omega_{2}, \ldots, \omega_{n}\right]^{T}$ and the output $y=x_{1}=$ $z_{1}$. So, we have

$$
\dot{V}_{n} \leq-\sum_{i=1}^{n} C_{i} z_{i}^{2}+\frac{\gamma^{2}}{2}\|\omega\|_{2}^{2}-\frac{1}{2}\|y\|_{2}^{2} \leq \frac{1}{2}\left(\gamma^{2}\|\omega\|_{2}^{2}-\|y\|_{2}^{2}\right) .
$$

Based on dissipation theory, the robust controller (18) makes the closed-loop system dissipative with the uncertain disturbance, and the supply rate $s(\omega, y)=\gamma^{2}\|\omega\|_{2}^{2}-\|y\|_{2}^{2}$. Furthermore, the finite-gain $L_{2}$ stability of the closed-loop system is concluded in the following theorem. 
Theorem 7. Consider the strict-feedback system with disturbance (8). There exists the smooth robust controller (18), such that the closed-loop system is finite-gain $L_{2}$ stable and the $L_{2}$ gain is no more than $\gamma$. Moreover, when the disturbance input $\omega=\left[\omega_{1}, \omega_{2}, \ldots, \omega_{n}\right]^{T}=0$, the closed-loop system is asymptotically stable.

Proof. From (20), we have $\dot{V}=\dot{V}_{n} \leq(1 / 2)\left(\gamma^{2}\|\omega\|_{2}^{2}-\|y\|_{2}^{2}\right)$. Integrating it yields the following inequality:

$$
V(x(\tau))-V(x(0)) \leq \frac{1}{2} \int_{0}^{\tau}\left(\gamma^{2}\|\omega\|_{2}^{2}-\|y\|_{2}^{2}\right) d t
$$

Due to $V(x) \geq 0$, we obtain

$$
\int_{0}^{\tau}\|y\|_{2}^{2} d t \leq \gamma^{2} \int_{0}^{\tau}\|\omega\|_{2}^{2} d t+2 V(x(0)) .
$$

Taking the square roots and using the inequality $\sqrt{a^{2}+b^{2}} \leq a+b$ for nonnegative numbers $a$ and $b$, one obtains

$$
\left\|y_{\tau}\right\|_{L_{2}} \leq \gamma\left\|\omega_{\tau}\right\|_{L_{2}}+\sqrt{2 V(x(0))} .
$$

Therefore, based on Definition 4, It is known that the system is finite-gain $L_{2}$ stable and the gain from disturbance input to system output is no more than $\gamma$.

Moreover, when $\omega=0$, from (20) we have

$$
\dot{V}=\dot{V}_{n} \leq \frac{\gamma^{2}}{2}\|\omega\|^{2}-\frac{1}{2}\|y\|^{2} \leq-\frac{1}{2}\|y\|^{2} \leq 0,
$$

and the storage function $V=(1 / 2) \sum_{i=1}^{n} z_{i}^{2}$ is positive definite. According to the invariance principle [1], we need to find $S=$ $\left\{X_{n} \in \mathbb{R}^{n} \mid \dot{V}=0\right\}$. Note that

$$
\dot{V}=0 \Longrightarrow y=0 \Longrightarrow x_{1}=0 \text {. }
$$

Hence, $S=\left\{X_{n} \in \mathbb{R}^{n} \mid x_{1}=0\right\}$. Let $X_{n}(t)$ be a solution that belongs identically to $S$. From the system (8), we can deduce that

$x_{1} \equiv 0 \Longrightarrow \dot{x}_{1} \equiv 0 \Longrightarrow x_{2} \equiv 0 \Longrightarrow \dot{x}_{2} \equiv 0 \Longrightarrow \cdots \Longrightarrow x_{n} \equiv 0$.

Therefore, the only solution that can stay identically in $S$ is the trivial solution $X_{n}(t) \equiv 0$. Thus, the closed-loop system is asymptotically stable in the case of $\omega=0$.

Remark 8. About the proposed method, there are the following opinions.

(1) This method integrates the idea of dissipation property into the recursive design. In the frame of dissipation theory, by using the feedback domination technology, the unknown disturbance is decoupled from the known state and gathered together to satisfy the dissipation inequality, which leads to the result of disturbance rejection and finite-gain $L_{2}$ stability.
(2) In the previous research of lower-triangular system (1), some assumption is needed for the uncertain disturbance, such as boundary condition and functional constraint. In this paper, the assumption is relaxed to be bounded, which can ensure that the closed-loop system is finite-gain $L_{2}$ stable. On the other hand, just for there is no constraint about the uncertain disturbance, the result is only finite-gain $L_{2}$ stable.

(3) It is interesting to note that the external disturbance $\omega_{i}$ can be generalized to more general uncertainties $\Delta_{i}\left(X_{i}, t\right)$, which satisfies the condition $\left|\Delta_{i}\left(X_{i}, t\right)\right| \leq$ $\omega_{i}(t) \phi_{i}\left(X_{i}\right)$, with $\phi_{i}\left(X_{i}\right)$ being a known smooth function and $\omega_{i}(t)$ being the unknown bound. The proposed method is still applicable for the more general case.

\section{Robust Controller Design of Lower-Triangular Systems}

The robust control law of lower-triangular systems (1) is designed in this section. Similarly, for $i=1,2, \ldots, n$, the design parameter $C_{i}>0$.

Step 1. Let $z_{1}=x_{1}$. Due to Assumption 1, the storage function is constructed as $V_{1}=z_{1}^{p_{1}-p_{1}+2} /\left(p_{1}-p_{1}+2\right)=z_{1}^{2} / 2$, and its derivative is

$$
\dot{V}_{1}=z_{1} \dot{z}_{1}=z_{1}\left(f_{1}\left(x_{1}\right)+x_{2}^{p_{1}}\right)+z_{1} \omega_{1} .
$$

Similar to the design of strict-feedback systems, one obtains:

$$
\dot{V}_{1} \leq z_{1}\left[x_{2}^{p_{1}}+f_{1}\left(x_{1}\right)+\left(\frac{1}{2}+\frac{n}{2 \gamma^{2}}\right) z_{1}\right]+\frac{\gamma^{2}}{2 n} \omega_{1}^{2}-\frac{z_{1}^{2}}{2} .
$$

Owing to (7), for any positive real number $\sigma>0$, let $b=\sigma$, $a=\left|z_{1}\left[f_{1}+\left((1 / 2)+\left(n / 2 \gamma^{2}\right)\right) z_{1}\right]\right|$, and $m=p_{1}+1$. We have

$$
\left|z_{1}\left[f_{1}+\left(\frac{1}{2}+\frac{n}{2 \gamma^{2}}\right) z_{1}\right]\right| \leq \sigma+z_{1}^{p_{1}+1} \rho_{1}\left(z_{1}\right)
$$

where $\rho_{1}\left(z_{1}\right)=\left(1 /\left(p_{1}+1\right)\right)\left[p_{1} /\left(p_{1}+1\right) \sigma\right]^{p_{1}}\left(f_{1}+z_{1} / 2+\right.$ $\left.n z_{1} / 2 \gamma^{2}\right)^{p_{1}+1} \geq 0$.

Substituting (29) into (28) yields

$$
\dot{V}_{1} \leq z_{1} x_{2}^{p_{1}}+z_{1}^{p_{1}+1} \rho_{1}\left(z_{1}\right)+\frac{\gamma^{2}}{2 n} \omega_{1}^{2}-\frac{z_{1}^{2}}{2}+\sigma
$$

Paying attention to Assumption 1, we design the smooth virtual control as

$$
\alpha_{2}=-z_{1}\left(C_{1}+1+\rho_{1}\left(z_{1}\right)\right)^{1 / p_{1}} .
$$

Taking the controller (31) into (30) results in

$$
\dot{V}_{1} \leq-\left(C_{1}+1\right) z_{1}^{p_{1}+1}+z_{1}\left(x_{2}^{p_{1}}-\alpha_{2}^{p_{1}}\right)+\frac{\gamma^{2}}{2 n} \omega_{1}^{2}-\frac{z_{1}^{2}}{2}+\sigma .
$$


Step $k$. Through $k-1$ steps, a group of virtual controllers are $z_{1}=x_{1}, z_{2}=x_{2}-\alpha_{2}, \ldots, z_{k-1}=x_{k-1}-\alpha_{k-1}$, and the storage function is $V_{k-1}=\sum_{i=1}^{k-1}\left(z_{i}^{p_{1}-p_{i}+2} /\left(p_{1}-p_{i}+2\right)\right)$, whose derivative is

$$
\begin{aligned}
\dot{V}_{k-1} \leq & -\sum_{i=1}^{k-1} C_{i} z_{i}^{p_{1}+1}-z_{k-1}^{p_{1}+1}+z_{k-1}^{p_{1}-p_{k-1}+1}\left(x_{k}^{p_{k-1}}-\alpha_{k}^{p_{k-1}}\right) \\
& +\frac{\gamma^{2}}{2} \sum_{j=1}^{k-1} \frac{k-j}{n+1-j} \omega_{j}^{2}-\frac{z_{1}^{2}}{2}+(k-1) \sigma .
\end{aligned}
$$

Let $z_{k}=x_{k}-\alpha_{k}$, and consider the storage function $V_{k}=$ $V_{k-1}+z_{k}^{p_{1}-p_{k}+2} /\left(p_{1}-p_{k}+2\right)$; its derivative is obtained as

$$
\begin{aligned}
\dot{V}_{k}= & \dot{V}_{k-1}+z_{k}^{p_{1}-p_{k}+1} \dot{z}_{k} \\
\leq & -\sum_{i=1}^{k-1} C_{i} z_{i}^{p_{1}+1}-z_{k-1}^{p_{1}+1}+z_{k-1}^{p_{1}-p_{k-1}+1}\left(x_{k}^{p_{k-1}}-\alpha_{k}^{p_{k-1}}\right) \\
+ & \frac{\gamma^{2}}{2} \sum_{j=1}^{k} \frac{k+1-j}{n+1-j} \omega_{j}^{2}-\frac{z_{1}^{2}}{2}+(k-1) \sigma \\
+ & z_{k}^{p_{1}-p_{k}+1}\left[x_{k+1}^{p_{k}}+f_{k}\left(X_{k}\right)+\frac{n+1-k}{2 \gamma^{2}} z_{k}^{p_{1}-p_{k}+1}\right. \\
& +\frac{z_{k}^{p_{1}-p_{k}+1}}{2 \gamma^{2}} \sum_{m=1}^{k-1}(n+1-m)\left(\frac{\partial \alpha_{k}}{\partial x_{m}}\right)^{2} \\
& \left.\quad-\sum_{m=1}^{k-1} \frac{\partial \alpha_{k}}{\partial x_{m}}\left(f_{m}+x_{m+1}^{p_{m}}\right)\right]
\end{aligned}
$$

Due to Young's inequality, there exists the smooth function $\bar{\rho}_{k}\left(z_{1}, \ldots, z_{k}\right)$, such that

$$
\left|z_{k-1}^{p_{1}-p_{k-1}+1}\left(x_{k}^{p_{k-1}}-\alpha_{k}^{p_{k-1}}\right)\right| \leq z_{k-1}^{p_{1}+1}+z_{k}^{p_{1}+1} \bar{\rho}_{k}\left(z_{1}, \ldots, z_{k}\right),
$$

where

$$
\begin{aligned}
& \bar{\rho}_{k}\left(z_{1}, \ldots, z_{k}\right) \\
& =\frac{p_{k-1}}{p_{1}+1}\left(2^{p_{k-1}-2} p_{k-1}\right){ }^{\left(p_{1}+1\right) / p_{k-1}} \\
& \quad \times\left[\frac{2\left(p_{1}-p_{k-1}+1\right)}{p_{1}+1}\right]^{\left(p_{1}-p_{k-1}+1\right) / p_{k-1}} \\
& +\frac{1}{p_{1}+1}\left[\left(1+2^{p_{k-1}-2}\right) p_{k-1}\right. \\
& \quad \times\left(\frac{2 p_{1}}{p_{1}+1}\right)^{p_{1}},
\end{aligned}
$$

and the construction of $\bar{\rho}_{k}\left(z_{1}, \ldots, z_{k}\right)$ can refer to [19].
Then, we define a smooth function as follows:

$$
\begin{aligned}
D_{k}\left(z_{1}, \ldots, z_{k}\right)= & f_{k}\left(X_{k}\right)+\frac{n+1-k}{2 \gamma^{2}} z_{k}^{p_{1}-p_{k}+1} \\
& +\frac{z_{k}^{p_{1}-p_{k}+1}}{2 \gamma^{2}} \sum_{m=1}^{k-1}(n+1-m)\left(\frac{\partial \alpha_{k}}{\partial x_{m}}\right)^{2} \\
& -\sum_{m=1}^{k-1} \frac{\partial \alpha_{k}}{\partial x_{m}}\left(f_{m}+x_{m+1}^{p_{m}}\right) .
\end{aligned}
$$

Similar to Step 1, we obtain

$\left|z_{k}^{p_{1}-p_{k}+1} D_{k}\left(z_{1}, \ldots, z_{k}\right)\right| \leq \sigma+z_{k}^{p_{1}+1} \rho_{k}\left(z_{1}, \ldots, z_{k}\right)$.

Taking (35) and (38) into (34) yields

$$
\begin{aligned}
\dot{V}_{k} \leq & -\sum_{i=1}^{k-1} C_{i} z_{i}^{p_{1}+1}+z_{k}^{p_{1}-p_{k}+1} x_{k+1}^{p_{k}}+z_{k}^{p_{1}+1}\left(\bar{\rho}_{k}(\cdot)+\rho_{k}(\cdot)\right) \\
& +\frac{\gamma^{2}}{2} \sum_{j=1}^{k} \frac{k+1-j}{n+1-j} \omega_{j}^{2}-\frac{z_{1}^{2}}{2}+k \sigma .
\end{aligned}
$$

Design the following virtual control law

$$
\alpha_{k+1}=-z_{k}\left(C_{k}+1+\bar{\rho}_{k}\left(z_{1}, \ldots, z_{k}\right)+\rho_{k}\left(z_{1}, \ldots, z_{k}\right)\right)^{1 / p_{k}},
$$

which renders

$$
\begin{aligned}
\dot{V}_{k} \leq & -\sum_{i=1}^{k} C_{i} z_{i}^{p_{1}+1}-z_{k}^{p_{1}+1}+z_{k}^{p_{1}-p_{k}+1}\left(x_{k+1}^{p_{k}}-\alpha_{k+1}^{p_{k}}\right) \\
& +\frac{\gamma^{2}}{2} \sum_{j=1}^{k} \frac{k+1-j}{n+1-j} \omega_{j}^{2}-\frac{z_{1}^{2}}{2}+k \sigma .
\end{aligned}
$$

Step $n$. In the end, for the storage function, $V=V_{n}=$ $\sum_{i=1}^{n}\left(z_{i}^{p_{1}-p_{i}+2} /\left(p_{1}-p_{i}+2\right)\right)$, where $z_{n}=x_{n}-\alpha_{n}$. There exists the smooth controller

$$
\begin{aligned}
u\left(z_{1}, \ldots, z_{n}\right)=-z_{n}( & C_{n}+\bar{\rho}_{n}\left(z_{1}, \ldots, z_{n}\right) \\
& \left.+\rho_{n}\left(z_{1}, \ldots, z_{n}\right)\right)^{1 / p_{n}}
\end{aligned}
$$

such that

$$
\dot{V}_{n} \leq-\sum_{i=1}^{n} C_{i} z_{i}^{p_{1}+1}+\frac{\gamma^{2}}{2} \sum_{j=1}^{n} \omega_{j}^{2}-\frac{z_{1}^{2}}{2}+n \sigma .
$$

Similar to Section 3, we finish this section with the following theorem.

Theorem 9. Consider the lower-triangular system with disturbance (1). There exists the smooth robust controller (42), such that the closed-loop system is finite-gain $L_{2}$ stable and the $L_{2}$ gain is no more than $\gamma$.

Proof. The proof of this theorem is similar to Theorem 7 , which is omitted. 


\section{Example and Application}

Example 1. Consider the stabilization problem of a lowertriangular system as follows:

$$
\begin{gathered}
\dot{x}_{1}=x_{1}^{2}+x_{2}^{3}+\omega_{1} \\
\dot{x}_{2}=x_{1} x_{2}^{2}+x_{2} e^{x_{2}}+u^{3}+\omega_{2} \\
y=x_{1},
\end{gathered}
$$

where $\omega_{1}$ and $\omega_{2}$ are bounded unknown disturbance.

Firstly, let $z_{1}=x_{1}$, and construct the storage function $V_{1}=z_{1}^{2} / 2$. According to the design steps, the virtual control is obtained as

$$
\alpha_{2}=-z_{1}\left(C_{1}+1+\rho_{1}\left(z_{1}\right)\right)^{1 / 3},
$$

where $\rho_{1}\left(z_{1}\right)=\left(27 / 256 \sigma^{3}\right)\left(x_{1}^{2}+x_{1} / 2+x_{1} / \gamma^{2}\right)^{4}$, and the positive parameters $\sigma$ and $\gamma$ can be adjusted according to the requirements.

Secondly, let $z_{2}=x_{2}-\alpha_{2}$ and $V_{2}=V_{1}+z_{2}^{2} / 2$. Based on the design process, the robust controller is taken as

$$
u=-z_{2}\left(C_{2}+\rho_{2}\left(z_{1}, z_{2}\right)+\bar{\rho}_{2}\left(z_{1}, z_{2}\right)\right)^{1 / 3},
$$

where

$$
\begin{gathered}
\rho_{2}\left(z_{1}, z_{2}\right)=\frac{1}{4}\left(\frac{3}{4 \sigma}\right)^{3}\left[x_{1} x_{2}^{2}+x_{2} e^{x_{2}}+\frac{z_{2}}{\gamma^{2}}\left(\frac{\partial \alpha_{2}}{\partial x_{1}}\right)^{2}\right. \\
\left.-\frac{\partial \alpha_{2}}{\partial x_{1}}\left(x_{1}^{2}+x_{2}\right)\right]^{4} \\
\bar{\rho}_{2}\left(z_{1}, z_{2}\right)=\frac{9}{2} \sqrt[3]{3}+\frac{27}{32}\left[9\left(C_{1}+1+\rho_{1}\left(z_{1}\right)\right)^{2 / 3}\right]^{4} .
\end{gathered}
$$

According to Theorem 9, it is known that the solutions of the system are globally bounded and the closed-loop system is finite-gain $L_{2}$ stable.

In the simulation, the system parameters are given as follows: $C_{1}=1, C_{2}=1, \sigma=2$, and $\gamma=2$. When the disturbances are $\omega_{1}=5, \omega_{2}=4 \sin t$, the state curves are shown as Figure 1, and the control law is in Figure 2. From them, we know that the closed-loop system is finite-gain $L_{2}$ stable and the gain is no more than $\gamma=2$.

Example 2. Consider one machine connected to an infinite bus system which is shown in Figure 3. The dynamic model of synchronous generator can be described as follows:

$$
\begin{gathered}
\dot{\delta}=\omega-\omega_{0} \\
\dot{\omega}=-\frac{D}{H}\left(\omega-\omega_{0}\right)+\frac{\omega_{0}}{H}\left(P_{m}-P_{e}\right)+\omega_{1} \\
\dot{P}_{m}=-\frac{P_{m}}{T_{H \Sigma}}+\frac{P_{m 0}}{T_{H \Sigma}}+\frac{C}{T_{H \Sigma}} \mu+\omega_{2},
\end{gathered}
$$

where

$$
P_{e}=\frac{E_{q}^{\prime} V_{s}}{X_{d \Sigma}^{\prime}} \sin \delta+\frac{V_{s}^{2}}{2}\left(\frac{X_{d \Sigma}^{\prime}-X_{q \Sigma}}{X_{d \Sigma}^{\prime} X_{q \Sigma}}\right) \sin 2 \delta,
$$

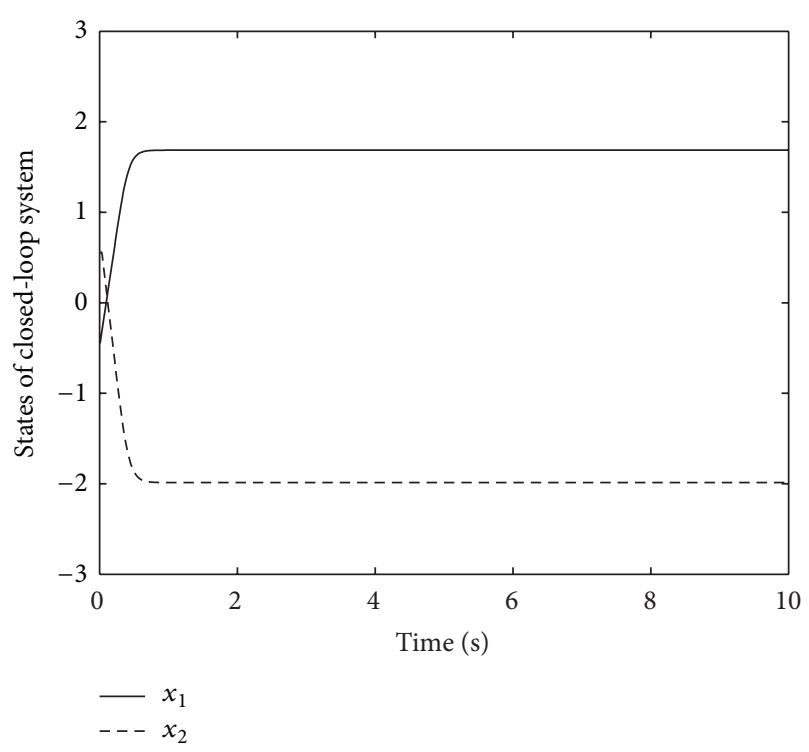

FIGURE 1: State response curves.

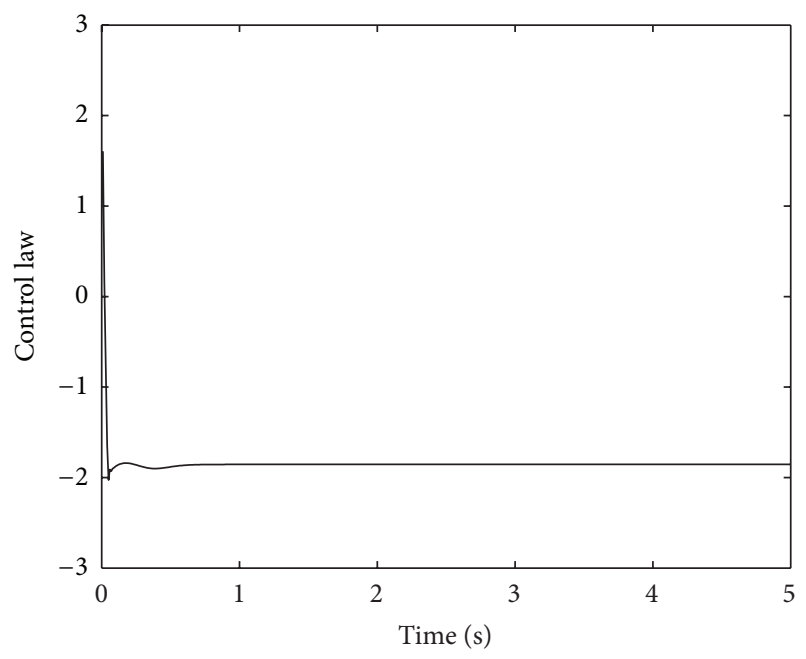

FIgURE 2: Curve of control law.

$\delta$ is the power angle; $\delta_{0}$ is the operating point of power angle; $\omega$ is the relative speed; $\omega_{0}$ is the synchronous machine speed; $P_{m}$ is the mechanical input power; $P_{m 0}$ is the operating point of mechanical input power; $P_{e}$ is the electromagnetic power; $\mu$ is the steam-valving controller; the uncertain disturbances $\omega_{1}$ and $\omega_{2}$ are bounded.

Let $\left(\delta_{0}, \omega_{0}, P_{m 0}\right)$ be the operating point, and define state variables by $x_{1}=\delta-\delta_{0}, x_{2}=\omega-\omega_{0}$, and $x_{3}=P_{m}-P_{m 0}$; then, system (48) and (49) is represented by

$$
\begin{gathered}
\dot{x}_{1}=x_{2} \\
\dot{x}_{2}=-\frac{D}{H} x_{2}-\frac{\omega_{0}}{H} \Delta P_{e}+\frac{\omega_{0}}{H} x_{3}+\omega_{1} \\
\dot{x}_{3}=-\frac{1}{T_{H \Sigma}} x_{3}+\frac{C}{T_{H \Sigma}} \mu+\omega_{2}
\end{gathered}
$$




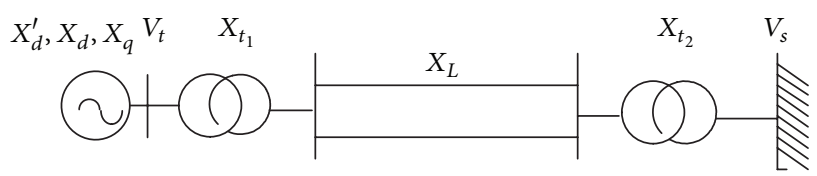

FIGURE 3: One-machine to infinite-bus circuit.

where

$$
\begin{aligned}
\Delta P_{e}= & \frac{E_{q}^{\prime} V_{s}}{X_{d \Sigma}^{\prime}}\left[\sin \left(\delta_{0}+x_{1}\right)-\sin \delta_{0}\right] \\
& +\frac{V_{s}^{2}}{2}\left(\frac{X_{d \Sigma}^{\prime}-X_{q \Sigma}}{X_{d \Sigma}^{\prime} X_{q \Sigma}}\right)\left[\sin 2\left(\delta_{0}+x_{1}\right)-\sin 2 \delta_{0}\right] .
\end{aligned}
$$

From the model (50) and (51), we know that this is a strict-feedback system. According to the design process in Section 3, the robust controller is obtained as follows:

$$
\begin{aligned}
\mu=T_{H \Sigma}\{- & C_{3} z_{3}+\frac{x_{3}}{T_{H \Sigma}}-z_{2}-\frac{z_{2}}{2 \gamma^{2}} \\
\times & {\left[1+3\left(\frac{\partial \alpha_{3}}{\partial x_{1}}\right)^{2}+2\left(\frac{\partial \alpha_{3}}{\partial x_{2}}\right)^{2}\right] } \\
& \left.+\frac{\partial \alpha_{3}}{\partial x_{1}} x_{2}+\frac{\partial \alpha_{3}}{\partial x_{2}}\left(-\frac{D}{H} x_{2}-\frac{\omega_{0}}{H} \Delta P_{e}+x_{3}\right)\right\},
\end{aligned}
$$

where $z_{1}=x_{1}, z_{2}=x_{2}-\alpha_{2}, z_{3}=x_{3}-\alpha_{3}, \alpha_{2}=-\left(C_{1}+1 / 2+\right.$ $\left.3 / 2 \gamma^{2}\right) z_{1}$,

$$
\begin{gathered}
\alpha_{3}=\frac{H}{\omega_{0}}\left[-C_{2} z_{2}+\frac{D}{H} x_{2}+\frac{\omega_{0}}{H} \Delta P_{e}-z_{1}-\frac{3 z_{2}}{2 \gamma^{2}}\left(\frac{\partial \alpha_{2}}{\partial x_{1}}\right)^{2}\right. \\
\left.-\frac{z_{2}}{\gamma^{2}}+\frac{\partial \alpha_{2}}{\partial x_{1}} x_{2}\right] .
\end{gathered}
$$

In order to verify the viability and effectiveness of the control law (52), the computer simulation is performed with the following parameters: $D=8, H=2, C=1, V_{s}=1$, $T_{H \Sigma}=0.35$, and $P_{m 0}=0.87455$ (see Figures 4, 5, 6, and 7).

In the presence of external disturbances $\omega_{1}=10$ and $\omega_{2}=15 \cos t$, the state responses and control law are shown in Figures 4 and 5, respectively. In the absence of disturbance, the simulation results are given in Figures 6 and 7. It is obvious that the proposed controller is effective for bounded disturbance and the closed-loop system can achieve finitegain $L_{2}$ stable. And, with the reduction of disturbance, the $L_{2}$ gain decreases. At last, when the disturbance vanishes, the closed-loop system is asymptotically stable as shown in Figure 6.

Moreover, in order to demonstrate the superiority of the proposed controller, we compare it with another robust

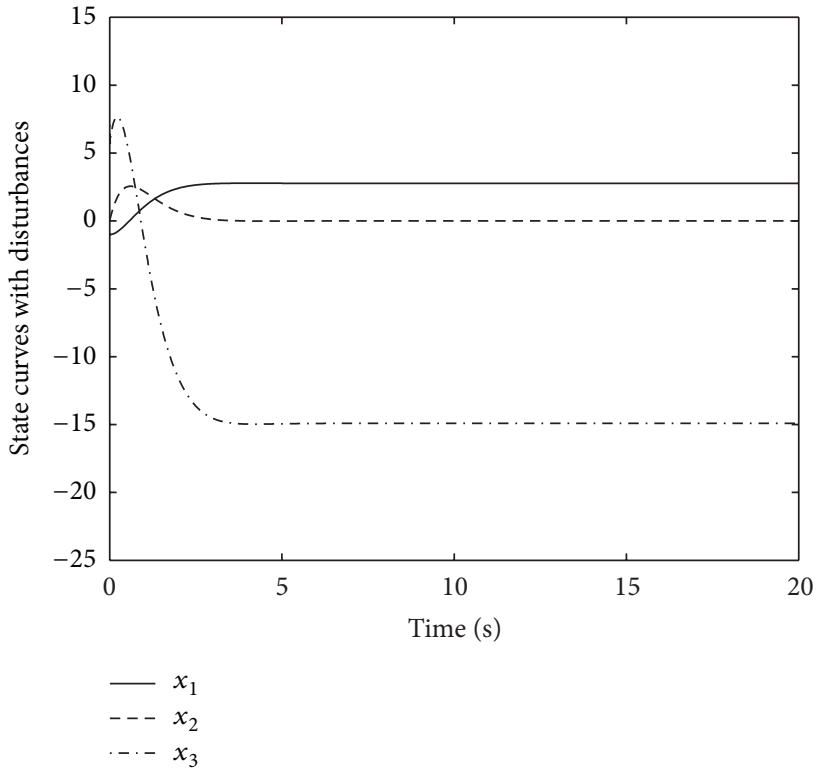

FIGURE 4: State curves with disturbances.

controller designed based on Hamiltonian function method [20].

Firstly, Hamilton energy function is constructed as

$$
\begin{aligned}
H= & \frac{E_{q}^{\prime} V_{s}}{X_{d \Sigma}^{\prime}}(1-\cos \delta)+\frac{V_{s}^{2}}{2}\left(\frac{X_{q \Sigma}-X_{d \Sigma}^{\prime}}{X_{d \Sigma}^{\prime} X_{q \Sigma}}\right) \cos ^{2} \delta \\
& +H \frac{(\Delta \omega)^{2}}{2 \omega_{0}}+\frac{1}{2}\left(P_{m}-P_{m 0}\right)^{2}+P_{m 0}(\pi-\delta) .
\end{aligned}
$$

Then, the model (48) in Hamilton form is rewritten into

$$
\left[\begin{array}{c}
\dot{\delta} \\
\Delta \dot{\omega} \\
\dot{P}_{m}
\end{array}\right]=\left[\begin{array}{ccc}
0 & \frac{\omega_{0}}{H} & 0 \\
-\frac{\omega_{0}}{H} & -\frac{D \omega_{0}}{H^{2}} & \frac{\omega_{0}}{H} \\
0 & 0 & -\frac{1}{T_{H \Sigma}}
\end{array}\right] \nabla H+\left[\begin{array}{c}
0 \\
0 \\
\frac{C}{T_{H \Sigma}}
\end{array}\right] \mu+\left[\begin{array}{c}
0 \\
\omega_{1} \\
\omega_{2}
\end{array}\right] .
$$

Based on Hamiltonian function method, the robust controller is designed as

$$
\mu=\mu_{0}-\Gamma G^{T} \nabla H=-\frac{T_{H \Sigma}}{C} \Delta \omega-\Gamma \frac{C}{T_{H \Sigma}}\left(P_{m}-P_{m 0}\right),
$$

where $\mu_{0}=-\left(T_{H \Sigma} / C\right) \Delta \omega$ is pre-feedback control and $\Gamma \in \mathbb{R}$ is design parameter.

Next, two methods are compared by the simulations. Figures 8,10 , and 12 are the controlled results with Hamiltonian function method; Figures 9, 11, and 13 are the controlled results with dissipation theory method.

In the absence of disturbance, from Figures 8 and 9, it is known that both of them are effective for the generator 


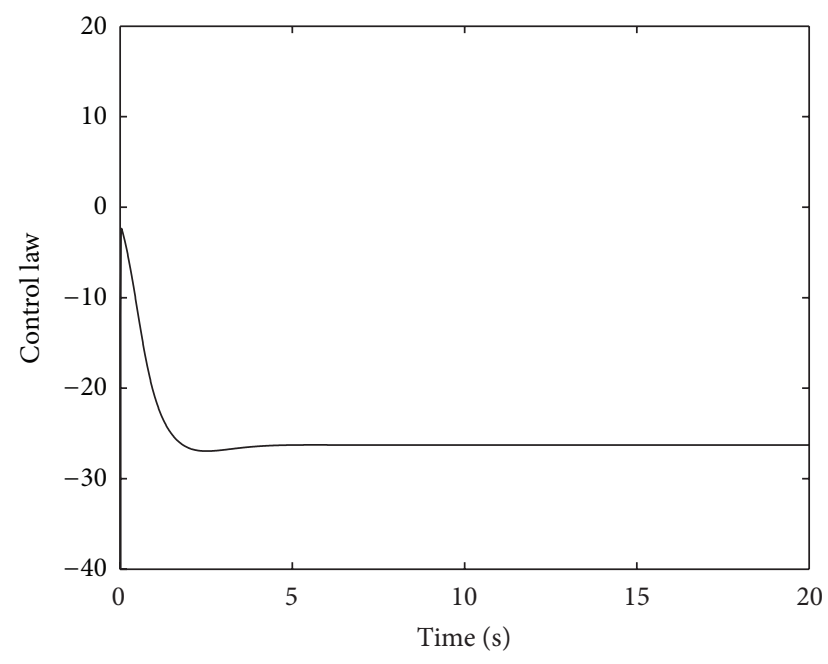

FIGURE 5: Control law with disturbances.

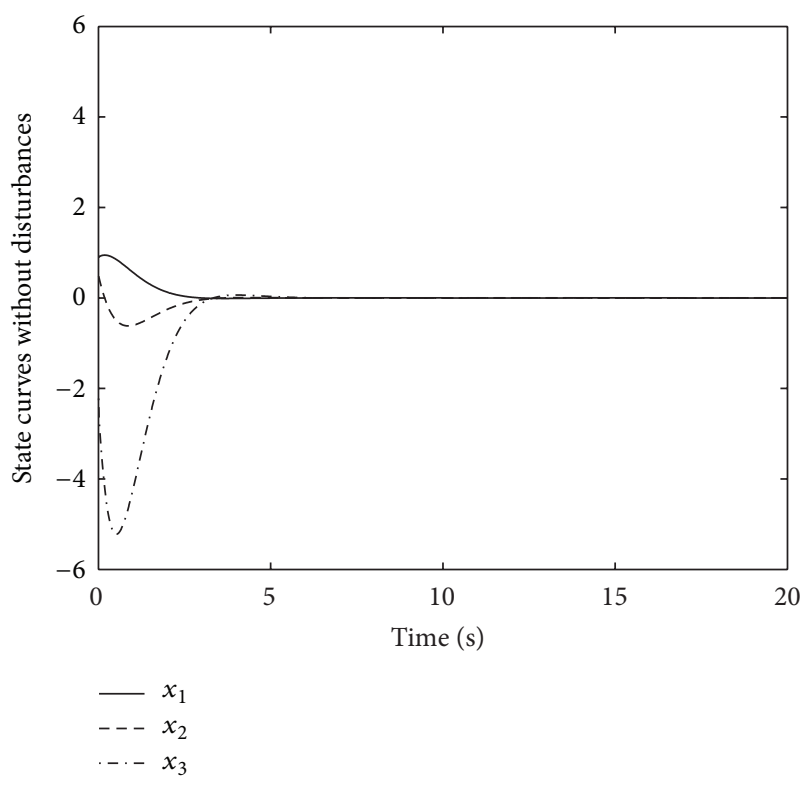

FIGURE 6: State curves without disturbances.

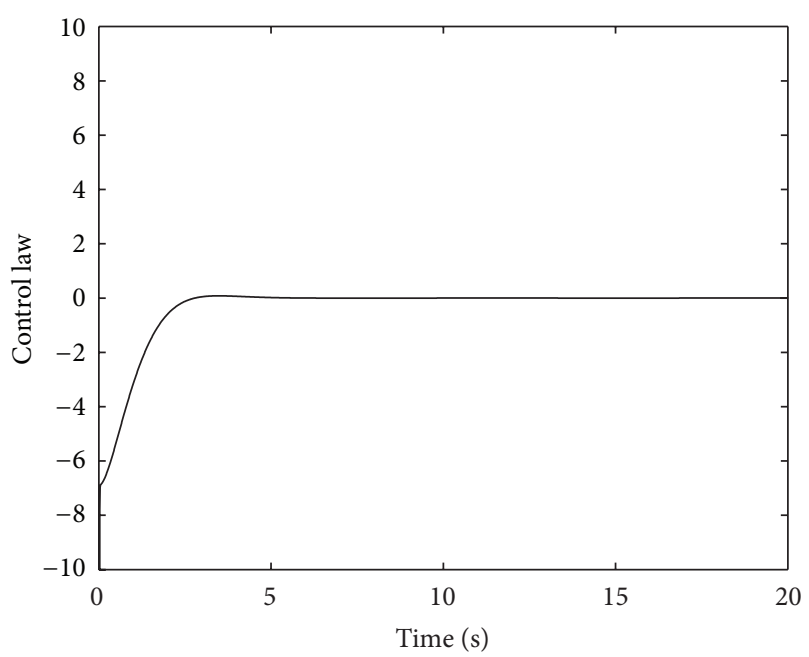

Figure 7: Control law without disturbances.

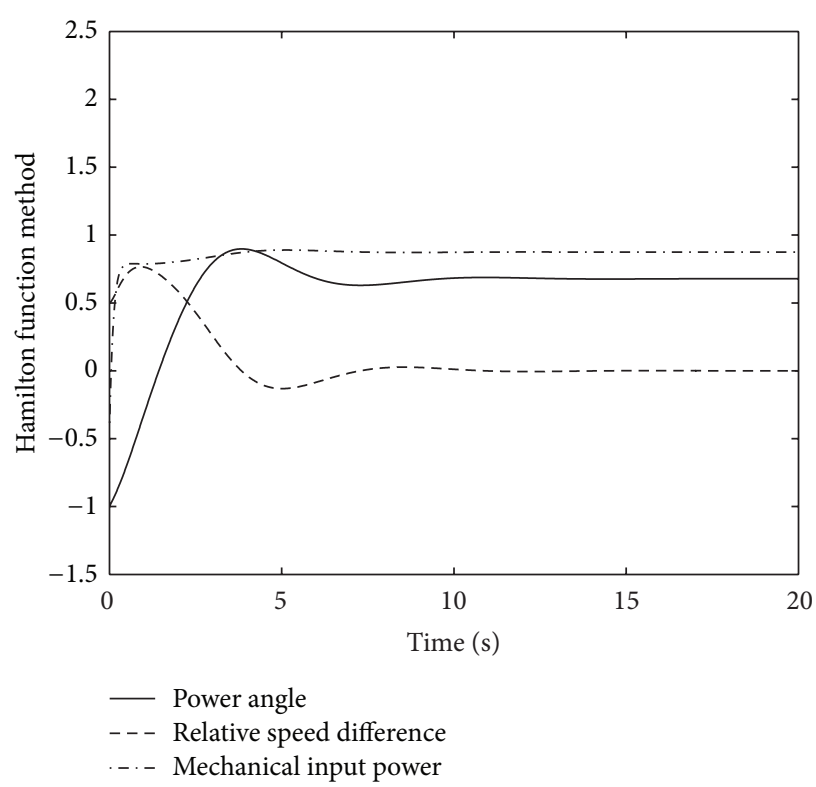

FIGURE 8: Response curves of Hamiltonian function method without disturbances.

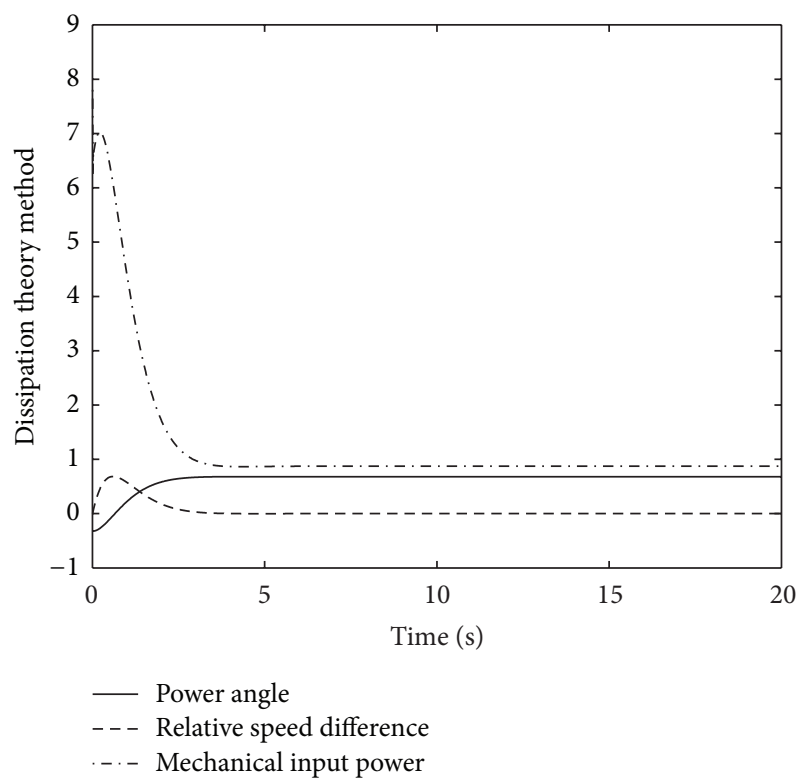

FIGURE 9: Response curves of dissipation theory method without disturbances.

system. From the dynamic process, it is seen that overshooting of Hamiltonian function method is less than that of dissipation theory method, and settling time of Hamiltonian function method is longer than that of dissipation. Then, in the presence of small disturbances with $\omega_{1}=1, \omega_{2}=1$, the simulation results are shown in Figures 10 and 11. It is shown that both of closed-loop systems are stable and the robust controller is effective. Next, with the enlargement of disturbances $\left(\omega_{1}=10, \omega_{2}=10\right)$, the response curves are shown in Figures 12 and 13, where the controlled system of Hamiltonian function method is unstable and the power 


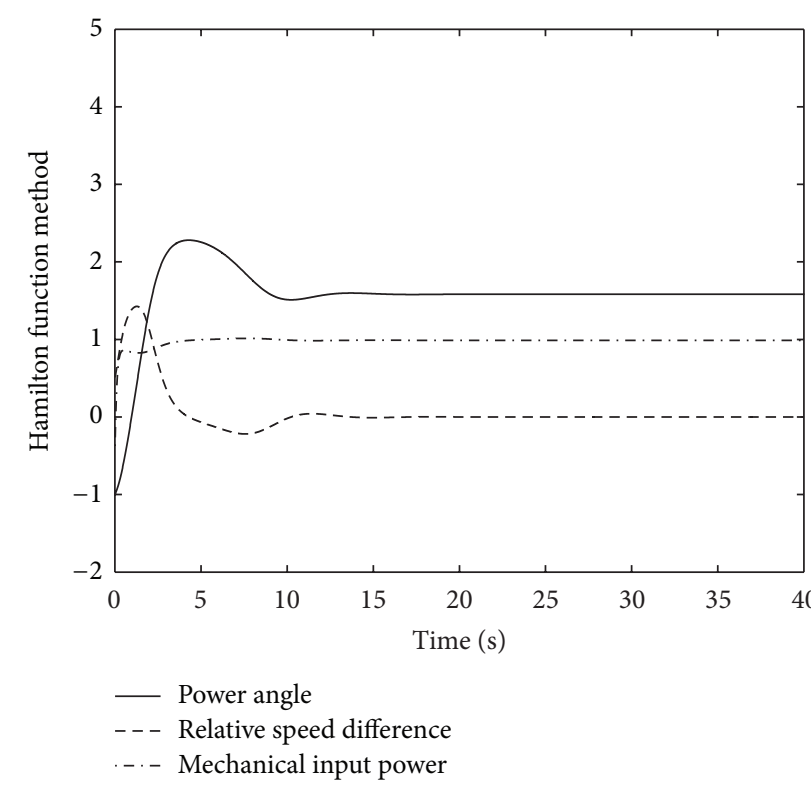

FIGURE 10: Response curves of Hamiltonian function method with small disturbances.

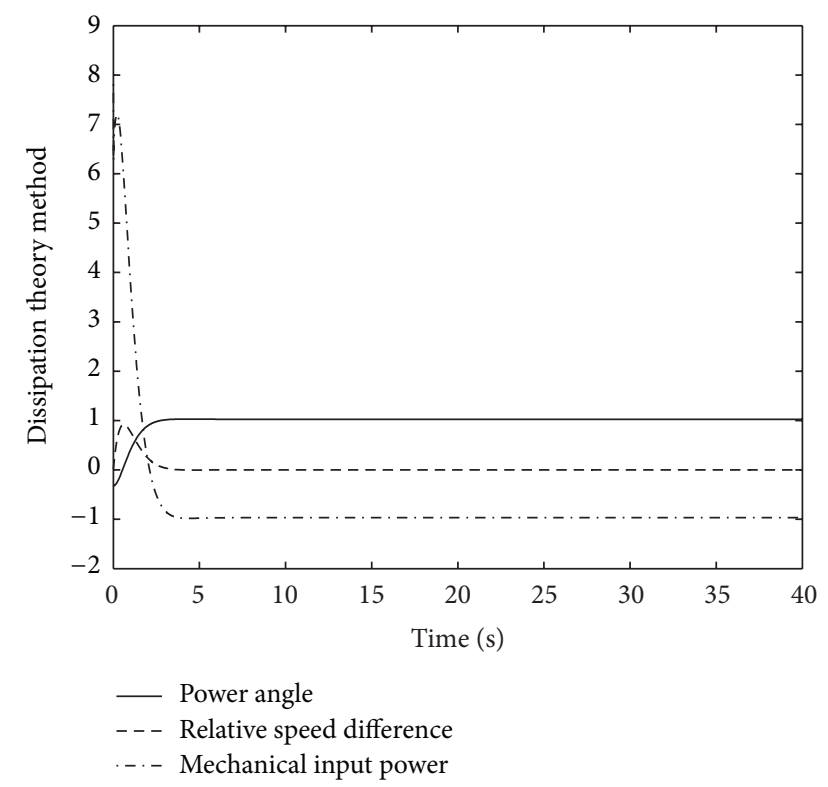

FIGURE 11: Response curves of dissipation theory method with small disturbances.

angle is out of control, while that of dissipation theory method is still stable, and the closed-loop system is $L_{2}$ stable.

In summary, based on Hamiltonian function method, the design process is concise, and the gotten controller (56) is simple, which is effective for the generator system in the presence of no disturbance or small disturbance. By using dissipation theory method, the construction of robust controller is relatively complex. However, it can guarantee that the closed-loop system is $L_{2}$ stable in all cases involving no disturbance, small disturbance, or large disturbance. Therefore, the proposed robust controller is more effective

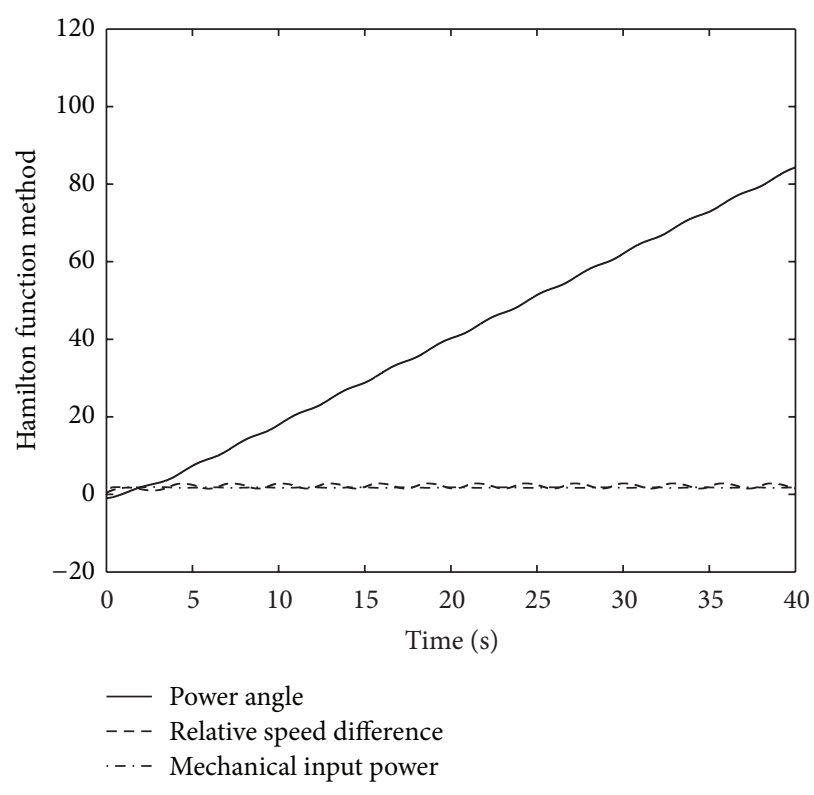

FIGURE 12: Response curves of Hamiltonian function method with large disturbances.

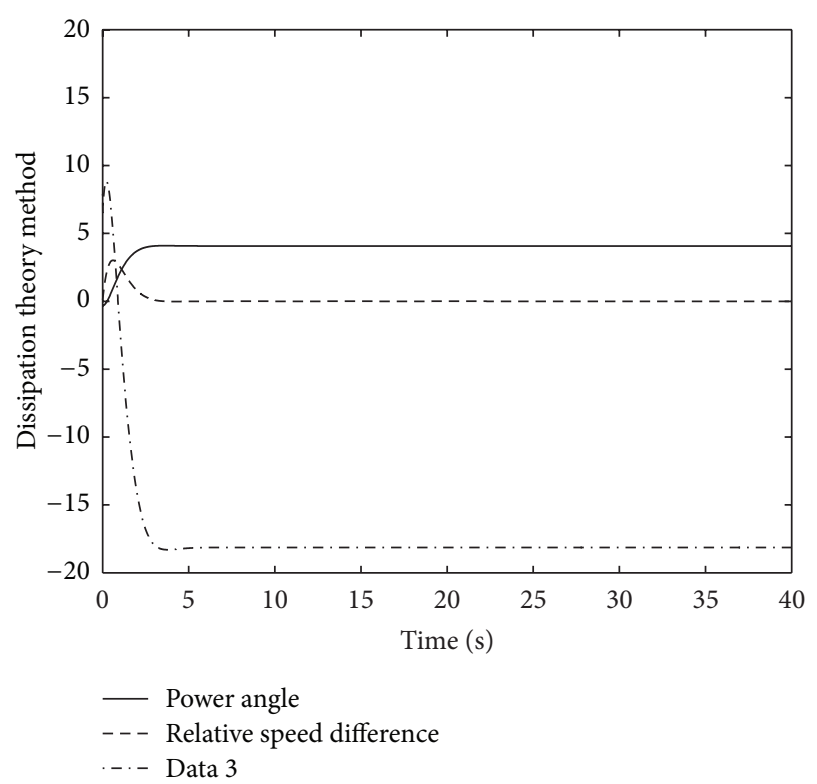

FIGURE 13: Response curves of dissipation theory method with large disturbances.

for the system with uncertain disturbance, especially for large disturbance.

\section{Conclusions}

In lower-triangular nonlinear systems, strict-feedback systems and high-order lower-triangular systems are two classes of main mathematical models. In this paper, the dissipationbased nonlinear controller is proposed to solve the robust control problem of these two systems. Uncertain disturbance 
is dominated by the supply rate, and the stability analysis is based on the storage function. The design method integrates the energy supply, energy storage, and energy dissipation into the recursive construction of robust controller. The simulations illustrate that the gotten controller is effective and has the advantage in regulating large disturbance. In the future research, the dissipation-based idea can be expanded to the robust controller design for more nonlinear systems.

\section{Acknowledgment}

This work is supported by the National Natural Science Foundation of China (Grant no. 51007019).

\section{References}

[1] H. K. Khalil, Nonlinear Systems, Prentice-Hall, Upper Saddle River, NJ, USA, 3rd edition, 2002.

[2] M. Krstic, I. Kanellakopoulos, and P. V. Kokotovic, Nonlinear and Adaptive Control Design, Wiley, New York, NY, USA, 1995.

[3] R. Sepulchre, M. Jankovic, and P. V. Kokotovic, Constructive Nonlinear Control, Springer, New York, NY, USA, 1997.

[4] W. Lin and C. Qian, "Adding one power integrator: a tool for global stabilization of high-order lower-triangular systems," Systems and Control Letters, vol. 39, no. 5, pp. 339-351, 2000.

[5] H. Chen, H.-B. Ji, B. Wang, and H.-S. Xi, "Coordinated passivation techniques for the dual-excited and steam-valving control of synchronous generators," IEE Proceedings: Control Theory and Applications, vol. 153, no. 1, pp. 69-73, 2006.

[6] K. D. Do, Z. P. Jiang, and J. Pan, "On global tracking control of a VTOL aircraft without velocity measurements," IEEE Transactions on Automatic Control, vol. 48, no. 12, pp. 2212-2217, 2003.

[7] Z.-G. Hou, A.-M. Zou, L. Cheng, and M. Tan, "Adaptive control of an electrically driven nonholonomic mobile robot via backstepping and fuzzy approach," IEEE Transactions on Control Systems Technology, vol. 17, no. 4, pp. 803-815, 2009.

[8] C. I. Byrnes and A. Isidori, "New results and examples in nonlinear feedback stabilization," Systems and Control Letters, vol. 12, no. 5, pp. 437-442, 1989.

[9] J. Tsinias, "Sufficient Lyapunov-like conditions for stabilization," Mathematics of Control, Signals, and Systems, vol. 2, no. 4, pp. 343-357, 1989.

[10] J. Tsinias, “Triangular systems: a global extension of the coronpraly theorem on the existence of feedback-integrator stabilisers," European Journal of Control, vol. 3, no. 1, pp. 37-46, 1997.

[11] J.-M. Coron and L. Praly, "Adding an integrator for the stabilization problem," Systems and Control Letters, vol. 17, no. 2, pp. 89104, 1991.

[12] S. Celikovsky and E. Aranda-Bricaire, "Constructive nonsmooth stabilization of triangular systems," Systems and Control Letters, vol. 36, no. 1, pp. 21-37, 1999.

[13] M. Tzamtzi and J. Tsinias, "Explicit formulas of feedback stabilizers for a class of triangular systems with uncontrollable linearization," Systems and Control Letters, vol. 38, no. 2, pp. 115126, 1999.

[14] W. Lin and C. Qian, "Adaptive regulation of high-order lowertriangular systems: an adding a power integrator technique," Systems and Control Letters, vol. 39, no. 5, pp. 353-364, 2000.

[15] L. Liu, Z. Chen, and J. Huang, "Global disturbance rejection of lower triangular systems with an unknown linear exosystem,"
IEEE Transactions on Automatic Control, vol. 56, no. 7, pp. 16901695, 2011.

[16] C. Wen, J. Zhou, Z. Liu, and H. Su, "Robust adaptive control of uncertain nonlinear systems in the presence of input saturation and external disturbance," IEEE Transactions on Automatic Control, vol. 56, no. 7, pp. 1672-1678, 2011.

[17] Y. Yu and Y. Zhong, "Semi-global robust output tracking for non-linear uncertain systems in strict-feedback form," IET Control Theory and Applications, vol. 6, no. 5, pp. 751-759, 2012.

[18] R. Wang, G. Jin, and J. Zhao, "Robust fault-tolerant control for a class of switched nonlinear systems in lower triangular form," Asian Journal of Control, vol. 9, no. 1, pp. 68-72, 2007.

[19] C. Qian and W. Lin, "Practical output tracking of nonlinear systems with uncontrollable unstable linearization," IEEE Transactions on Automatic Control, vol. 47, no. 1, pp. 21-36, 2002.

[20] Z. Xi, D. Cheng, Q. Lu, and S. Mei, "Nonlinear decentralized controller design for multimachine power systems using Hamiltonian function method," Automatica, vol. 38 , no. 3, pp. 527$534,2002$. 


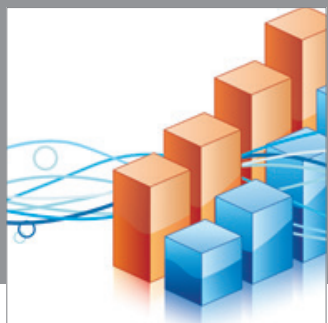

Advances in

Operations Research

mansans

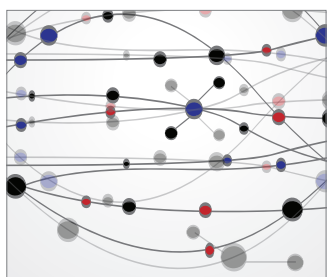

The Scientific World Journal
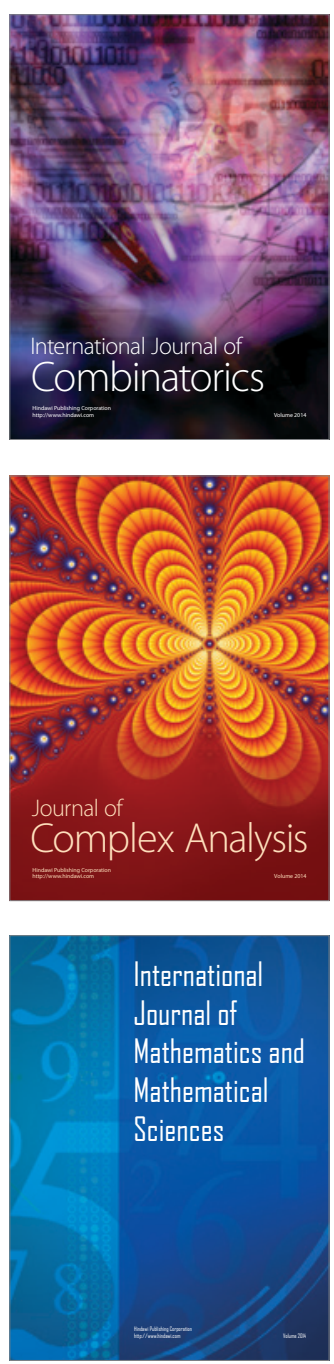
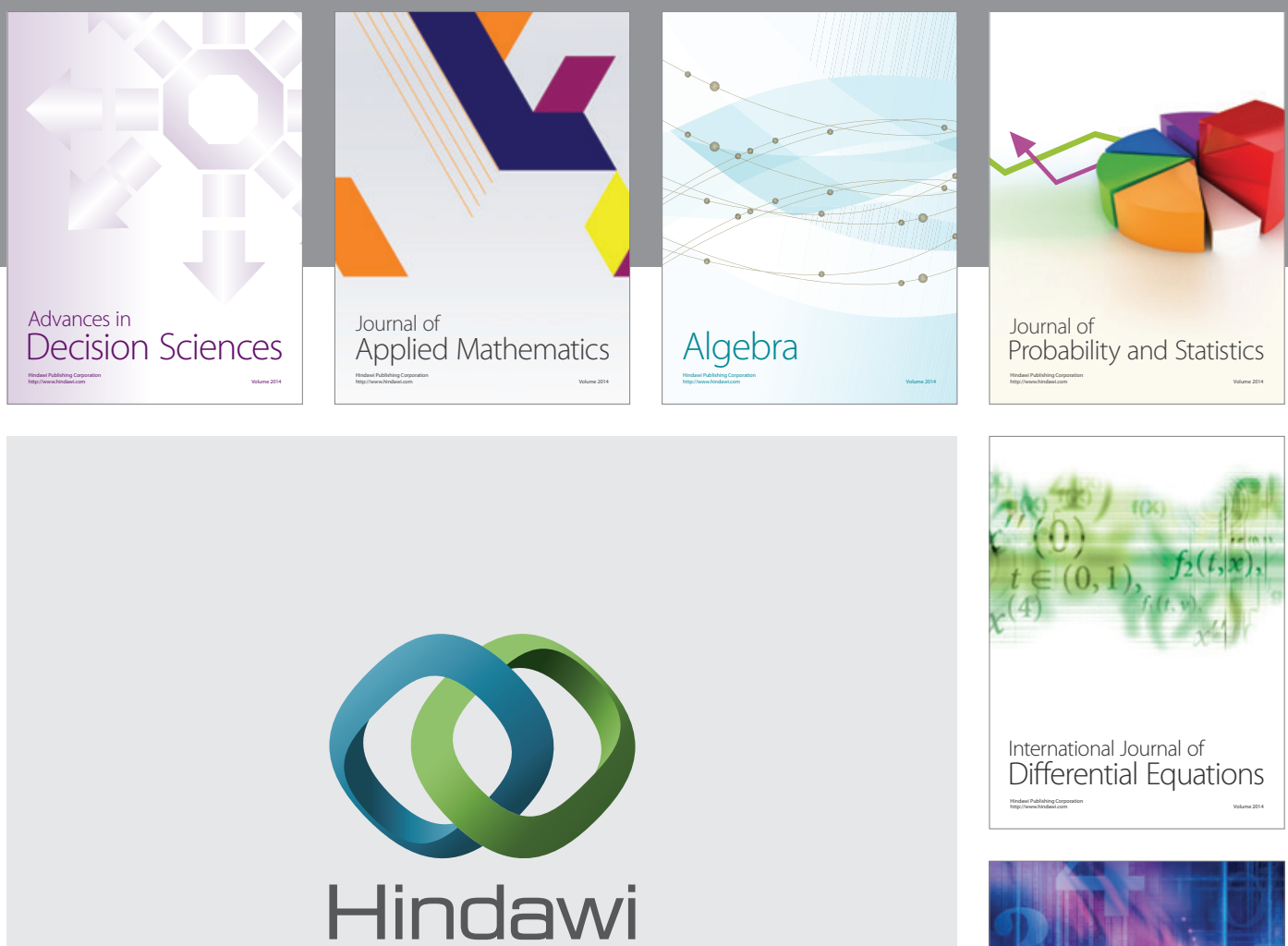

Submit your manuscripts at http://www.hindawi.com
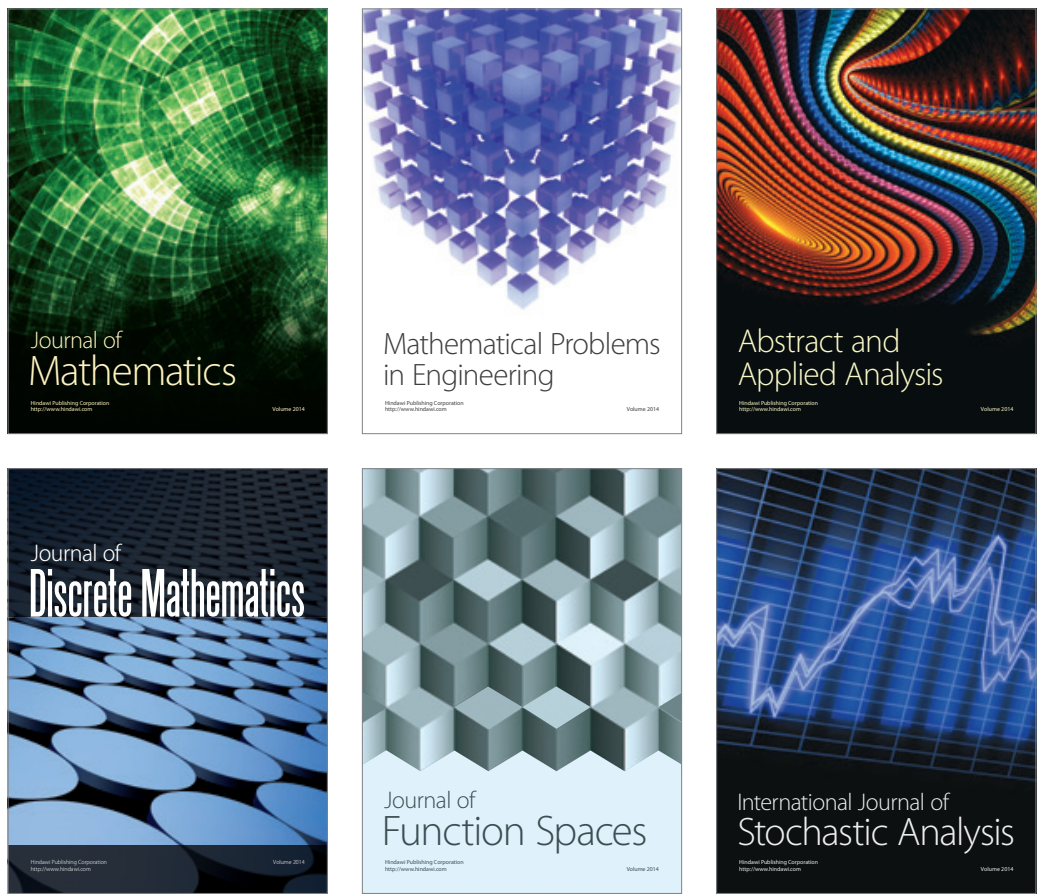

Journal of

Function Spaces

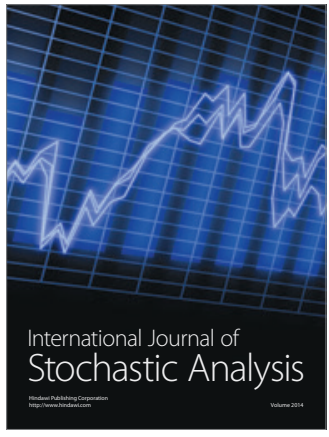

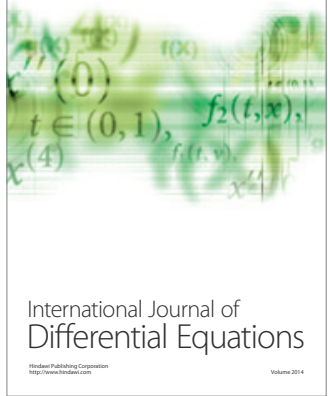
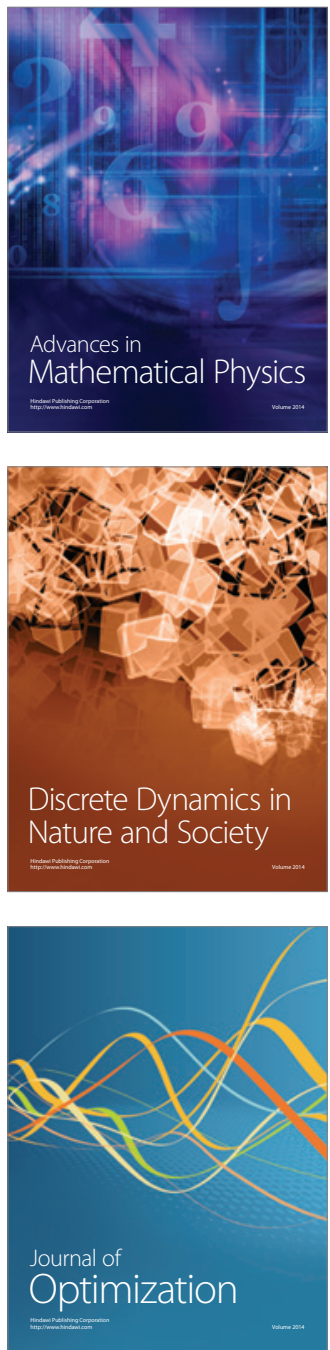